\title{
VLSI Implementation Using DWT For Image Compression and Image Fusion For Medical Applications
}

\author{
Aarti Shinde ${ }^{1}$, A K Pathrikar ${ }^{2}$ \\ Research scholar, Department of E\&TC, SPWE College, Aurangabad, India ${ }^{1}$ \\ Professor, Department of E\&TC, SPWE College, Aurangabad, India ${ }^{2}$
}

\begin{abstract}
Image fusion is a data fusion technology which keeps images as main research contents. It is techniques that integrate multi-images of the same scene from multiple image sensor data or integrate multi images of the same scene at different times from one image sensor. The fusion technique in medical images is useful for resourceful disease diagnosis purpose. For medical diagnosis, Computed Tomography (CT) provides the best information on denser tissue with less distortion. Magnetic Resonance Image (MRI) provides better information on soft tissue with more distortion. The image fusion algorithm based on Discrete Wavelet Transform which is faster developed was a multi-resolution analysis image fusion method in recent decade. Discrete Wavelet Transform has good time-frequency characteristics. The paper proposes that the method could extract useful information from source images to fused images so that clear images are obtained. In this paper, a hardware implementation of a image fusion system is proposed. Here using MATLAB we can convert images into pixel-format files and to observe simulation results. To implement this paper XPS \& VB are needed. Using XPS, first select hardware \& software components then by adding source and header files \& converting it into bit streams and download into FPGA, to obtain fused image. The input image can also be recovered by combining of fused image and the other input image.
\end{abstract}

Keywords: FPGA, MATLAB, Visual Basic, System

\section{INTRODUCTION}

Image fusion is the process of combining relevant fusions were classified into spatial domain fusion and information from two or more images into a single image. transform domain fusion. A Spatial domain fusion is Then the resultant image has more information than input directly applied on the source images which is reduces the images. Image fusion has become a topic of great interest signal to-noise ratio of the resultant image with simple to the engineers working in different fields. It's being used averaging technique but the spatial distortion still persists for medical applications, so as to get a better image. It's in the fused image. To improve on that in transform also being used in automotive industries to enhance the domain fusion, firstly the input images are decomposed vision of road, to observe a better image during a rainy into some levels based on transform coefficients. Then the weather. Due to the increasing number of high-resolution fusion technique is applied and the fusion decision map is images are available along with sensor technology obtained. using Inverse transformation on this decision development, image fusion is still important and easy map gives the fused image. The fused image includes all method to interpret the image data obtaining a more the details of the source images an and reduces the spatial suitable image for a variety of applications, such as visual distortion.

interpretation, satellite, , digital classification \& war-field. The main objective of medical imaging is to obtain a high resolution image with as much details as possible for the sake of diagnosis. MRI and CT imaging are of main concern for diagnostic purposes. Both techniques give special sophisticated characteristics of the organ to be imaged. So, it is expected that fusion of MRI and CT images of the same organ would result in an integrated image of much more information. Wavelet transform fusion is defined as considering the wavelet transforms of the two registered input images together with the fusion rule. Then, the inverse wavelet transform is computed, and the fused image is reconstructed. Generally the image fusion process can be carried out at various levels. Under this, in the pixel-level image fusion the fused images provided all relevant information present in original images without any inconsistencies. The pixel-level image

\section{RELATED WORK}

Over the years, multiple efforts have been made to develop new image fusion techniques. Additionally, efforts have been made to review and assess the image fusion qualities. However, there are still a large number of open issues that need to be resolved in this area. Jasiunas presented an image fusion system based on wavelet decomposition for unmanned airborne vehicles (UAVs). This is probably the first implementation developed on a reconfigurable platform also first investigation adaptive image fusion that makes use of dynamic reconfiguration to change the fusion algorithm as the UAV approaches an object of interest. Sims and Irvine presented an FPGA implementation using pyramid landed composition and subsequent fusion of dual video streams. This realization gives a design that can fuse dual video streams in gray 
scale video graphics array (VGA), with 30 frames/s in real time. Both hardware designs showed an improvement in speed performance compared to respective algorithms running on a typical PC, giving strong indications of the advantages of hardware against software implementation. Following are the some image fusion technologies having its own advantages and disadvantages A)Fusion using Principle Component Analysis (PCA): The PCA image fusion method simply uses the pixel values of all source images at each pixel location, and adding a weight factor to each pixel value, and takes an average of the weighted pixel values to produce the result for the fused image at the same pixel location. The optimal weighted factors are determined by the PCA technique. The PCA technique is useful for image encoding, image data compression, image enhancement B) Fusion using Laplacian pyramid method: The Laplacian pyramid fusion consists of an iterative process of calculating the Gaussian and Laplacian pyramids of each source image, fusing the Laplacian images at each pyramid level by selecting the pixel with the larger absolute value, combining the fused Laplacian pyramid with the combined pyramid expanded from the lower level, and then expanding the combined pyramids to the upper level C) Fusion using gradient pyramid method: A gradient pyramid is obtained by applying a set of 4 directional gradient filters (horizontal, vertical, and 2 diagonal) to the Gaussian pyramid at each level. At each level, these 4 directional gradient pyramids are combined together to obtain a combined gradient pyramid that is similar to a Laplacian pyramid. The gradient pyramid fusion is therefore the same as the fusion using the Laplacian pyramid method except replacing the Laplacian pyramid with the combined gradient pyramid.

\section{PROPOSED MODEL}

Image fusion has become a common term used within medical diagnostics and treatment. The term is used when multiple patient images are registered and overlaid or merged to provide additional information. Fused images may be created from multiple images from the same imaging modality, or by combining information from multiple modalities, such as magnetic resonance image (MRI), computed tomography (CT), positron emission tomography (PET), and single photon emission computed tomography (SPECT). The transform of a signal is just another form of representing the signal. The Wavelet Transform provides a time-frequency representation of the signal. It was developed to overcome the short coming of the Short Time Fourier Transform (STFT), which can also be used to analyze non-stationary signals. While STFT gives a constant resolution at all frequencies, the Wavelet Transform uses multi-resolution technique by which different frequencies are analyzed with different resolutions. In above block diagram the input we are taking is a CT scan image and MRI images in the form of text file. For the text file we convert the actual images using MATLAB GUI interface. The Wavelet Series is just a sampled version of CWT and its computation may consume significant amount of time and resources, depending on the resolution required.

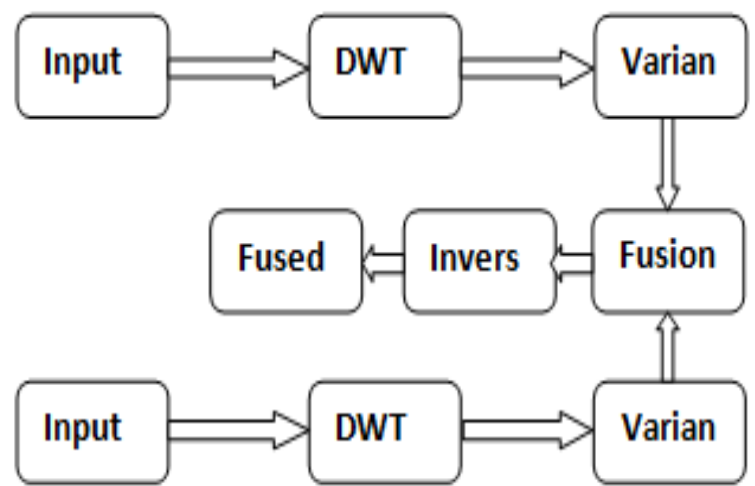

Fig.1 Proposed Authentication Block Diagram

The Discrete Wavelet Transform (DWT), which is based on sub-band coding, is found to yield a fast computation of Wavelet Transform. It is easy to implement and reduces the computation time and resources required. In CWT, the signals are analyzed using a set of basis functions which relate to each other by simple scaling and translation. In the case of DWT, a time-scale representation of the digital signal is obtained using digital filtering A) DWT Image Fusion The Discrete Wavelet Transform was developed to apply the wavelet transform to the digital world and Filter banks are used to approximate the behavior of the continuous wavelet transform. The signal is decomposed using a high-pass filter and a low-pass filter. The coefficients of these filters are computed by doing mathematical analysis and available in subsections 1. First register the input images (I1 and I2), which are going to be fused and corresponding pixels are aligned. 2. The Registered input images are decomposed into wavelet transformed images respectively, based on haar wavelet transformation (W). The transformed images with K-level decomposition will include one low-frequency portion (LL band) and $3 \mathrm{~K}$ high-frequency portions (LH bands, HL bands and $\mathrm{HH}$ bands). 3. The Transform coefficients of different portions are performed with a certain fusion rule. 4. Then by applying performing an inverse wavelet transform (W-1) based on the combined transform coefficients, the fused image (I) is constructed

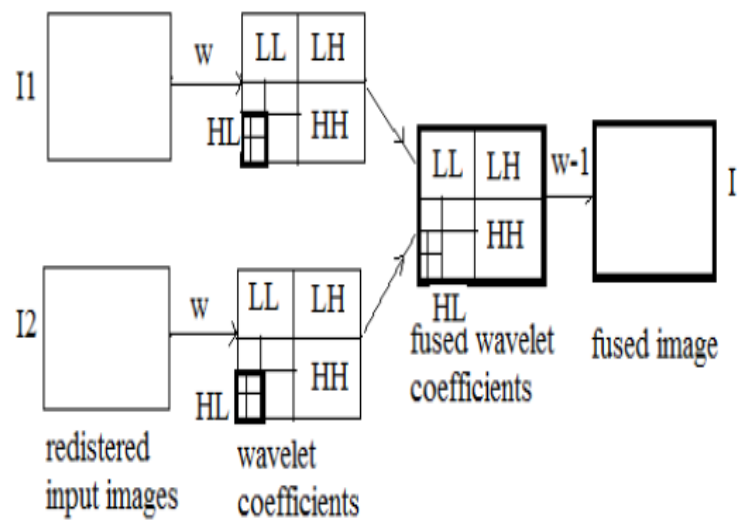

Fig.2. The Scheme of image fusion using wavelet transforms 
A) DWT Decomposition of Images

Filter banks decompose the signal into high- and lowfrequency components. The low-frequency component usually contains most of the frequency of the signal. This is called the approximation. In wavelet decomposing of an image, the decomposition is done row by row and then column by column. For instance, here is the procedure for an $\mathrm{N} \mathrm{x} \mathrm{M}$ image. Then filter each row and down-sample to obtain two $\mathrm{N} \times(\mathrm{M} / 2)$ images, they are $\mathrm{L}$ and $\mathrm{H}$ images. The formulas used to do find $\mathrm{H}$ and $\mathrm{L}$ is given below. $\mathrm{H}=$ odd-even $=\mathrm{F} 1 ; \mathrm{L}=$ even + round $(\mathrm{F} 1 / 2)$; Then filter each column and sub sample the filter output to obtain four $(\mathrm{N} / 2) \times(M / 2)$ images, by using the formulas shown in below. $\mathrm{LH}=$ odd - even $=\mathrm{F} 2 ; \mathrm{LL}=$ even + round $(\mathrm{F} 2 / 2)$; $\mathrm{HH}=$ odd - even $=\mathrm{F} 3 ; \mathrm{HL}=$ even + round $(\mathrm{F} 3 / 2)$; The four sub-images obtained as seen in Fig. 1, the one obtained by low-pass filtering the rows and columns is referred to as the LL image. Similarly LH, HL and HL are also formed. This process can be continued until the desired sub band structure is obtained. Here F1,F2 and F3 are taken as references.

\section{IMPLEMENTATION PROCESS}

The implementation steps of the image fusion using DWT is done as follows. MATLAB is used to convert input images into header files using GUI feature. Xilinx Platform Studio (XPS) is a part of an EDK system and it includes the XPS-GUI and all tools run by the GUI to process hardware \& software components within XPS.

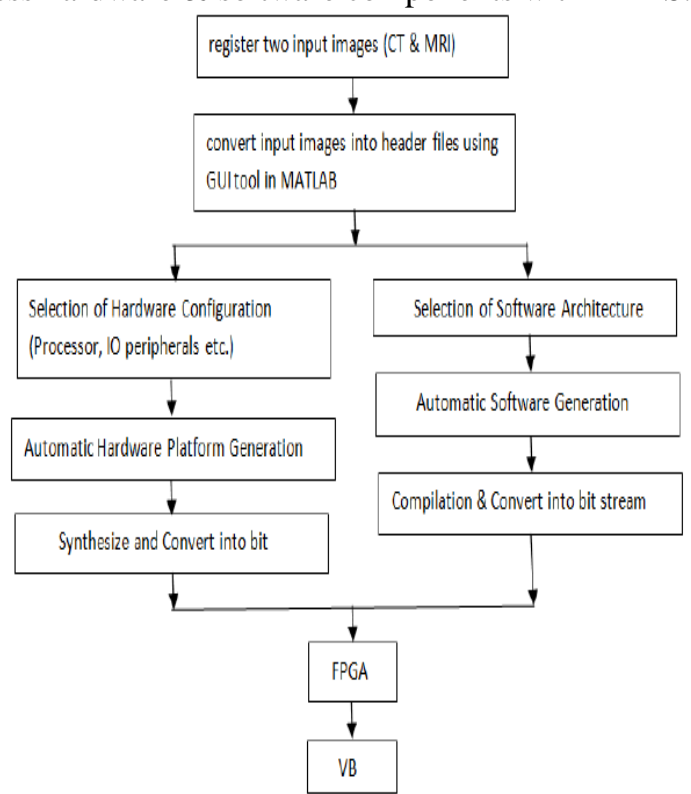

Fig .3 Flow chart for FPGA implementation of image fusion.

FPGA hardware is chosen because of it has some advantages like reconfigurability, low power dissipation, small size.

Step 1: First Select the Hardware configuration like processor, IO peripherals etc. The HDL codes are generated

Step 2: After that the selection of software architecture is started. It selects drivers, libraries etc. the header files of input images and source file (which consist of DWT, fusion and IDWT code written in the system-C language) are added and convert it into bit stream.

Step 3: Then download hardware and software architecture bit streams into FPGA and run it. The image output can't be shown in FPGA. For this purpose Visual Basic is used to observe the fused image output

\section{CONCLUSION}

In this paper, the hardware implementation of a fusion method that is suitable for remote sensing data has been presented. The DWT fusion method is configurable since it allows the user to control the correlation properties of the final fused color image. The hardware realization which is based on FPGA technology provides a fast, compact, and low-power solution for image fusion. The dedicated sections provide a detailed description of the methodology to transform the DWT fusion method in a hardware realizable process.

\section{ACKNOWLEDGEMENT}

It is a great pleasure for me to express my deep gratitude and sincere thanks to Prof.Moresh Mukhedkar and Prof. R. Satyanarayana, PG coordinator, Electronics and Communication Engineering Department, Dr.D.Y.Patil College of Engineering, Pune University, Pune. For their constant encouragement and valuable guidance during our research work.

\section{REFERENCES}

[1] A. Goshtaby and S. Nikolov, "Image fusion: Advances in the state of the art," Inf. Fusion, vol. 8, no. 2, pp. 114-118, Apr. 2007.

[2] T. Stathaki, Image Fusion: Algorithms and Applications. New York: Academic, 2008.

[3] R. S. Blum and Z. Liu, Eds., Multi-Sensor Image Fusion and Its Applications(Special Series on Signal Processing and Communications).New York: Taylor \& Francis, 2006

[4] C. Pohl and J. L. van Genderen, "Multisensor image fusion in remote sensing: Concepts, methods and applications," Int. J. Remote Sens., vol. 19,no. 5, pp. 823-854, 1998.

[5] V. Tsagaris, V. Anastassopoulos, and G. Lampropoulos, "Fusion of hyperspectral data using segmented PCT for enhanced color representation," IEEE Trans. Geosci.., vol. 43, no. 10, pp. 23652375,Oct. 2005.

[6] N. Jacobson, M. Gupta, and J. Cole, "Linear fusion of image sets for display," IEEE Trans. Geosci. Remote Sens., vol. 45, no. 10, pp. 3277-3288, Oct. 2007.

[7] K. Nagarajan, C. Krekeler, K. C. Slatton, and W. D. Graham, "A scalable approach data to estimate streamflow via a Bayesian network," IEEE Trans.., vol. 48, no. 10, pp. 3720-3732, Oct. 2010.

[8] G. Piella, "A general framework for multiresolution image fusion: From pixels to regions," Inf. Fusion, vol. 4, no. 4, pp. 259-280, Dec. 2003.

[9] C. Thomas, T. Ranchin, L. Wald, and J. Chanussot, "Synthesis of multispectral images to high spatial resolution: A critical review of fusion methods based on remote sensing physics," IEEE Trans. Geosci. Remote Sens., vol. 46, no. 5, pp. 1301-1312, May 2008.

[10] J. Tyo, A. Konsolakis, D. Diersen, and R. C. Olsen, "Principal components- based display strategy for spectral imagery," IEEE Trans. Geosci. Remote Sens., vol. 41, no. 3, pp. 708-718, Mar. 2003.

[11] W. Zhang and J. Kang, "QuickBird panchromatic and multi-spectral image fusion using wavelet packet transform," in Lecture Notes in Control and Information Sciences, vol. 344. Berlin, Germany: Springer-Verlag, 2006, pp. 976-981.

[12] V. Shah, N. Younan, and R. King, "An efficient pan-sharpening method via a combined adaptive PCA approach and contourlets," IEEETrans.Geosci.Remot 